\title{
Divisor Degree Energy of Some Graphs using Mat Lab Programme
}

\author{
K. Nagarajan, S. P. Kanniga Devi
}

\begin{abstract}
In this paper, we find the divisor degree matrix of wheel, fan, complete bipartite and splitting graph of star graph and generate their divisor degree energy. Also, we obtain the divisor degree matrix of a tree graphs such as path, star and comb graph and their divisor degree energy by using MAT LAB programme.
\end{abstract}

Keywords: Divisor degree matrix, Divisor Degree Energy

\section{INTRODUCTION} The divisor degree matrix $D D(G)$ and divisor degree energy $(D D E)$ of a graph are defined in [4].

The divisor degree energy of some standard graphs was found in [4], but it is very difficult to find the divisor degree energy for other graphs. So, we are using MAT LAB programme to find the divisor degree energy for few graphs and the output will be discussed in the following sections.

\section{THE DIVISOR DEGREE ENERGY OF SOME GRAPHS USING MAT LAB}

In this section, we obtain the divisor degree matrix and divisor degree energy of some graphs - wheel, fan, complete bipartite and splitting graph of star graph using MAT LAB programme.

Program 2.1. To calculate the divisor degree energy of wheel graph:

$\mathrm{n}=; \%$ Enter the number of vertices of a wheel graph

$\mathrm{DD}=$ zeros $(\mathrm{n})$;

for $\mathrm{i}=1: \mathrm{n}-1$

$\mathrm{DD}(\mathrm{i}, \mathrm{i}+1)=1$;

$\operatorname{DD}(\mathrm{i}+1, \mathrm{i})=1$;

$\operatorname{DD}(\mathrm{i}, \mathrm{n})=\mathrm{fix}((\mathrm{n}-1) / 3)$;

$\operatorname{DD}(\mathrm{n}, \mathrm{i})=\mathrm{fix}((\mathrm{n}-1) / 3)$;

end

$\mathrm{DD}(1, \mathrm{n}-1)=1$;

$\mathrm{DD}(\mathrm{n}-1,1)=1$;

DD

Eigenvaluesofwheelgraph=eig(DD)

$\mathrm{DDE}=\operatorname{sum}(\operatorname{abs}($ Eigenvaluesofwheelgraph $))$

Revised Manuscript Received on December 15, 2019.

Dr. K. Nagarajan, Associate Professor, Department of mathematics, Sri S. Ramasamy Naidu Memorial College, Sattur, India.

S. P. Kanniga Devi, Research Scholar, Department of mathematics, Sri S. Ramasamy Naidu Memorial College, Sattur, India.
Let $G$ be a simple graph with $n$ vertices and $m$ edges.

OUTPUT:

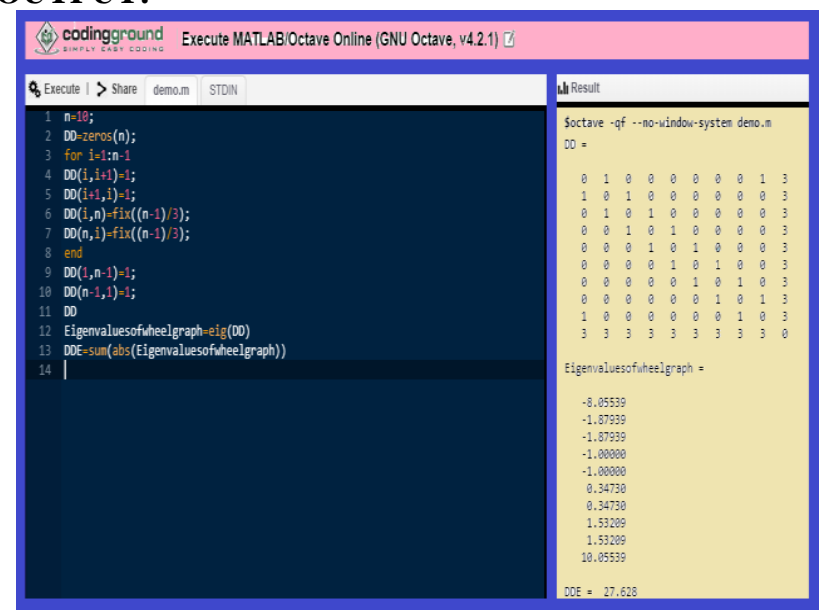

Figure 1: For $\mathbf{n}=10$, DDE of wheel graph.

Program 2.2. To calculate the divisor degree energy of a fan graph:

$\mathrm{n}=; \%$ Enter the number of vertices of fan graph

$\mathrm{DD}=$ zeros(n);

for $\mathrm{i}=1: \mathrm{n}-2$

$\operatorname{DD}(\mathrm{i}, \mathrm{i}+1)=1$;

$\operatorname{DD}(\mathrm{i}+1, \mathrm{i})=1$

$\operatorname{DD}(\mathrm{i}+1, \mathrm{n})=\operatorname{fix}((\mathrm{n}-1) / 3)$;

$\operatorname{DD}(\mathrm{n}, \mathrm{i}+1)=\operatorname{fix}((\mathrm{n}-1) / 3)$;

end

$\operatorname{DD}(1, \mathrm{n})=\mathrm{fix}((\mathrm{n}-1) / 2)$;

$\operatorname{DD}(\mathrm{n}, 1)=\mathrm{fix}((\mathrm{n}-1) / 2)$;

$\operatorname{DD}(\mathrm{n}-1, \mathrm{n})=\mathrm{fix}((\mathrm{n}-1) / 2)$;

$\operatorname{DD}(\mathrm{n}, \mathrm{n}-1)=\operatorname{fix}((\mathrm{n}-1) / 2)$;

DD

Eigenvaluesoffangraph=eig(DD)

DDE=sum(abs(Eigenvaluesoffangraph)

OUTPUT:

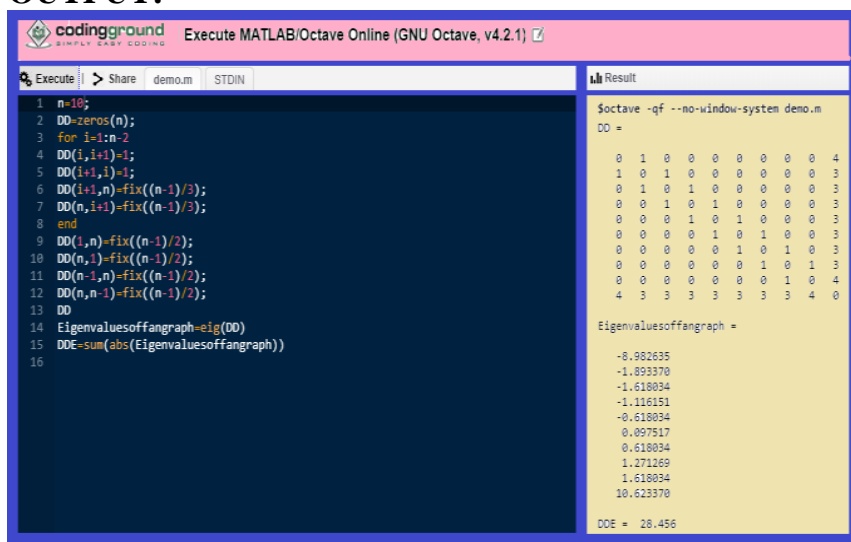

Figure 2: For $n=10$, DDE of fan graph

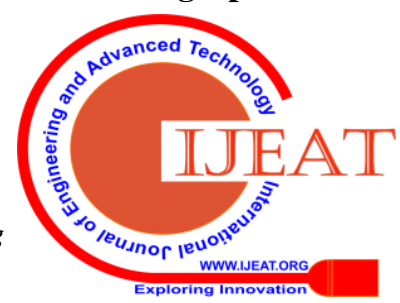




\section{Divisor Degree Energy of Some Graphs using Mat Lab Programme}

Program 2.3. To calculate the divisor degree energy of complete bipartite graph:

$\mathrm{p}=; \%$ Enter the number of vertices in a vertex set $\mathrm{X}$

$\mathrm{q}=; \%$ Enter the number of vertices in a vertex set $\mathrm{Y}$

$\mathrm{n}=\mathrm{p}+\mathrm{q}$;

$\mathrm{DD}=\mathrm{zeros}(\mathrm{n})$;

for $\mathrm{i}=1: \mathrm{p}$

for $\mathrm{j}=\mathrm{p}+1: \mathrm{n}$

$\operatorname{DD}(\mathrm{i}, \mathrm{j})=\mathrm{fix}(\mathrm{q} / \mathrm{p})$;

$\operatorname{DD}(\mathrm{j}, \mathrm{i})=\mathrm{fix}(\mathrm{q} / \mathrm{p})$;

end

end

DD

EigenvaluesofCompletebipartitegraph=eig(DD)

$\mathrm{DDE}=\operatorname{sum}(\mathrm{abs}($ EigenvaluesofCompletebipartitegraph $))$

\section{OUTPUT:}

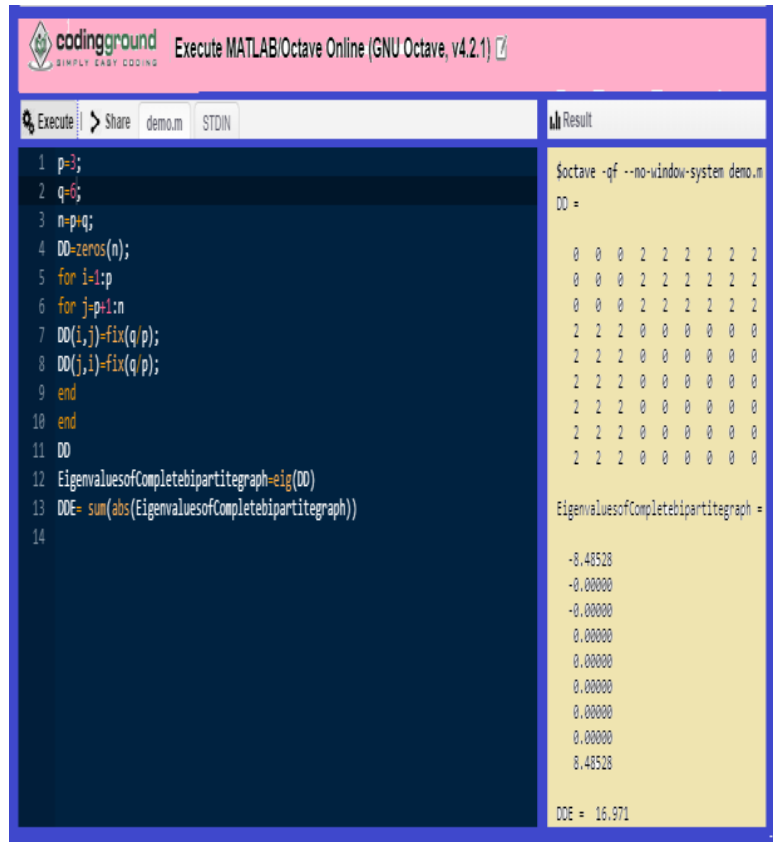

Figure 3: For n = 9, DDE of Complete bipartite graph.

Program 2.4. To calculate the divisor degree energy of splitting graph of a Star graph:

$\mathrm{p}=; \%$ Enter the number of vertices of star graph $(\mathrm{p}>2)$

$\mathrm{n}=2 * \mathrm{p}$;

$\mathrm{DD}=$ zeros $(\mathrm{n})$;

for $\mathrm{i}=1: \mathrm{p}-1$

for $\mathrm{j}=\mathrm{p}+1: 2 * \mathrm{p}-1$

$\mathrm{DD}(\mathrm{i}, \mathrm{p})=\mathrm{p}-1$;

$\mathrm{DD}(\mathrm{p}, \mathrm{i})=\mathrm{p}-1$;

$\operatorname{DD}(\mathrm{i}, 2 * \mathrm{p})=\mathrm{fix}((\mathrm{p}-1) / 2)$;

$\mathrm{DD}(2 * \mathrm{p}, \mathrm{i})=\mathrm{fix}((\mathrm{p}-1) / 2)$;

$\mathrm{DD}(\mathrm{j}, \mathrm{p})=2 *(\mathrm{p}-1)$;

$\mathrm{DD}(\mathrm{p}, \mathrm{j})=2 *(\mathrm{p}-1)$

end

end

DD

EigenvaluesofsplittinggraphofStargraph=eig(DD)

DDE=sum(abs(EigenvaluesofsplittinggraphofStargraph))

\section{OUTPUT:}

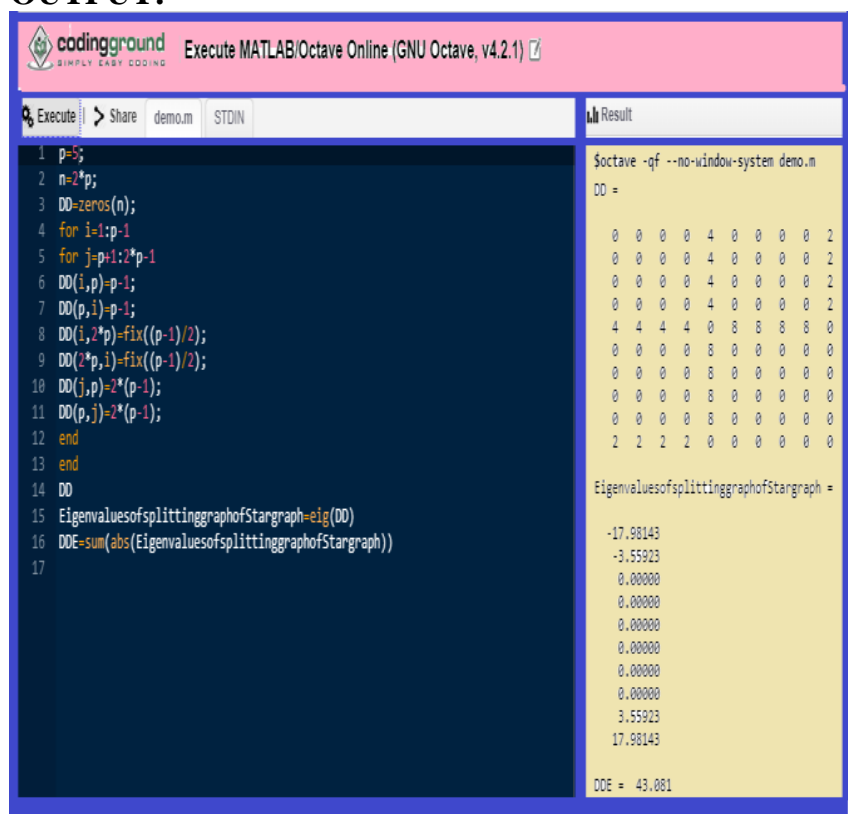

Figure 4: For $n=10$, DDE of Splitting of star graph.

Program 2.5. To calculate the divisor degree energy of thorn graph of complete graph:

$\mathrm{p}=; \%$ Enter the number of vertices of complete graph $\mathrm{n}=2 * \mathrm{p}$;

$\mathrm{DD}=\mathrm{zeros}(\mathrm{n})$;

for $\mathrm{i}=1: \mathrm{p}$

for $\mathrm{j}=1: \mathrm{p}$

$\mathrm{DD}(\mathrm{i}, \mathrm{i}+\mathrm{p})=\mathrm{p}$;

$\mathrm{DD}(\mathrm{i}+\mathrm{p}, \mathrm{i})=\mathrm{p}$;

$\operatorname{DD}(\mathrm{i}, \mathrm{j})=1$;

$\mathrm{DD}(\mathrm{j}, \mathrm{i})=1$;

$\mathrm{DD}(\mathrm{i}, \mathrm{i})=0$;

$\operatorname{DD}(\mathrm{j}, \mathrm{j})=0$;

end

end

DD

Eigenvaluesoftorngraphofcompletegraph=eig(DD)

$\mathrm{DDE}=\operatorname{sum}(\operatorname{abs}$ (Eigenvaluesoftorngraphofcompletegraph))

\section{OUTPUT:}

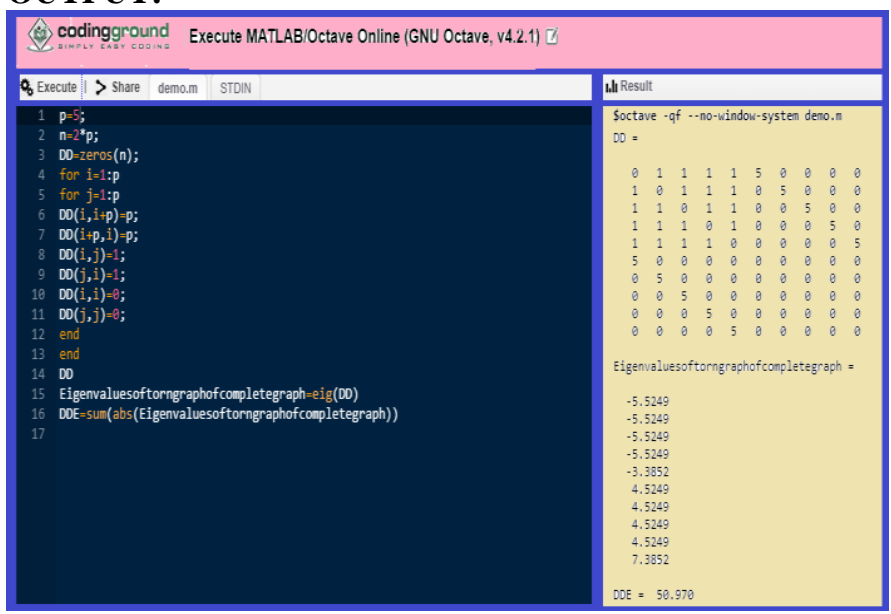

Figure 5: For $n=10$, DDE of thorn graph of complete graph. 
Program 2.6. To calculate the divisor degree energy of friendship graph:

$\mathrm{p}=; \%$ Enter the number of copies of $\mathrm{K} 3$

$\mathrm{n}=2 * \mathrm{p}+1$

$\mathrm{DD}=\mathrm{zeros}(\mathrm{n})$;

for $\mathrm{i}=1: \mathrm{p}$

for $\mathrm{j}=\mathrm{i}+\mathrm{p}: \mathrm{n}-1$

$\operatorname{DD}(2 * \mathrm{i}-1,2 * \mathrm{i})=1$

$\mathrm{DD}(2 * \mathrm{i}, 2 * \mathrm{i}-1)=1$;

$\mathrm{DD}(\mathrm{i}, \mathrm{n})=\mathrm{p}$;

$\mathrm{DD}(\mathrm{n}, \mathrm{i})=\mathrm{p}$;

$\mathrm{DD}(\mathrm{j}, \mathrm{n})=\mathrm{p}$;

$\mathrm{DD}(\mathrm{n}, \mathrm{j})=\mathrm{p}$;

end

end

DD

DD1 $=$ DD $*$ DD

Eigenvaluesoffriendshipgraph=eig(DD)

DDE=sum(abs(Eigenvaluesoffriendshipgraph))

\section{OUTPUT:}

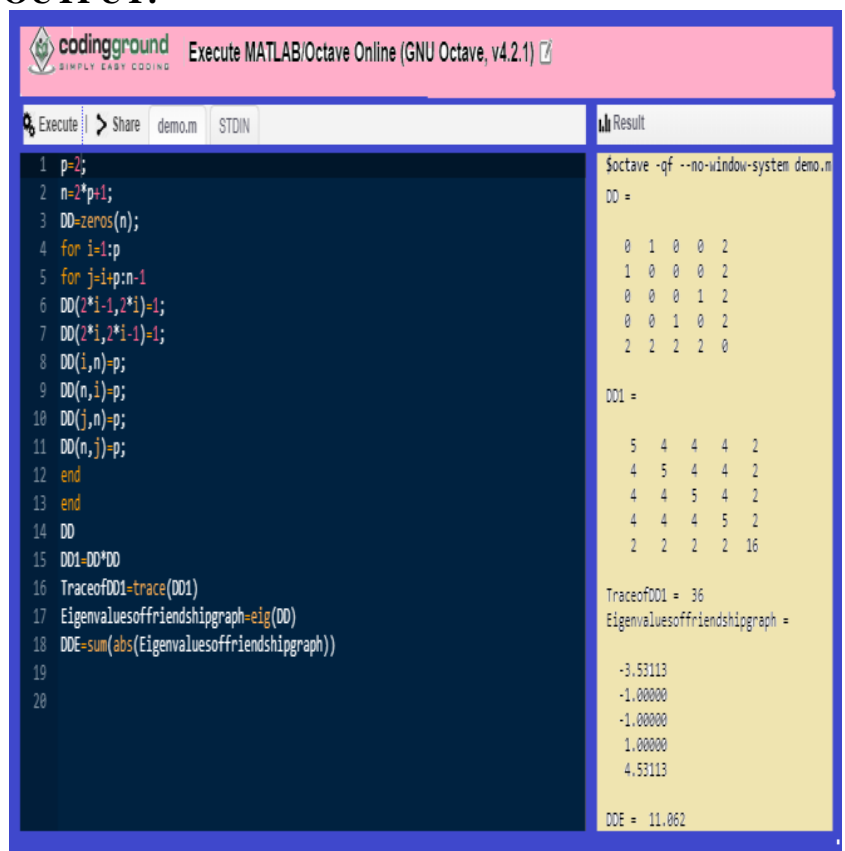

Figure 6: For n = 5, DDE of friendship graph.

Program 2.7. To calculate the divisor degree energy of ladder graph:

$\mathrm{p}=; \%$ Enter the number of vertices of path graph

$\mathrm{n}=2 * \mathrm{p}$

$\mathrm{DD}=\mathrm{zeros}(\mathrm{n})$;

for $\mathrm{i}=1: \mathrm{n}-1$

for $\mathrm{j}=1: \mathrm{p}-1$

$\mathrm{DD}(\mathrm{i}, \mathrm{i}+1)=1$;

$\mathrm{DD}(\mathrm{i}+1, \mathrm{i})=1$;

$\mathrm{DD}(\mathrm{p}-\mathrm{j}, \mathrm{p}+\mathrm{j}+1)=1$;

$\operatorname{DD}(p+j+1, p-j)=1$;

end

end

DD

$\mathrm{DD} 1=\mathrm{DD} * \mathrm{DD}$

TraceofDD1 $=$ trace $(\mathrm{DD} 1)$

Eigenvaluesofladdergraph=eig(DD)

DDE $=$ sum(abs(Eigenvaluesofladdergraph $))$
TraceofDD1 $=$ trace $(\mathrm{DD} 1)$

\section{OUTPUT:}

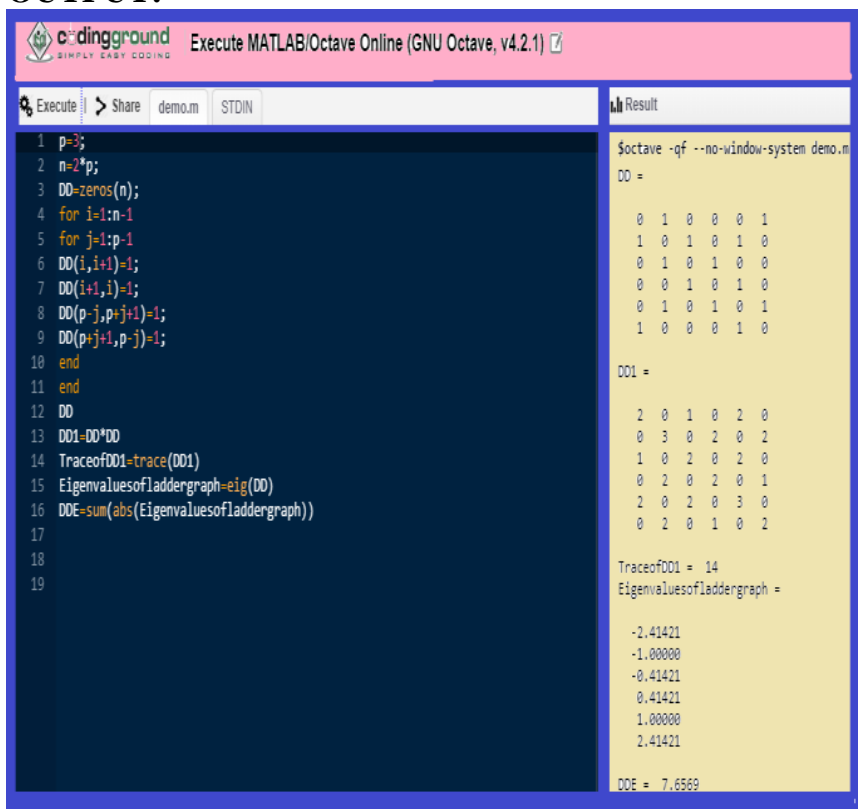

Figure 7: For n = 6, DDE of ladder graph.

Program 2.8. To calculate the divisor degree energy of path graph:

$\mathrm{n}=; \%$ Enter the number of vertices of path graph $(\mathrm{n}>2)$

$\mathrm{DD}=$ zeros $(\mathrm{n})$;

for $\mathrm{i}=1: \mathrm{n}-2$

$\mathrm{DD}(\mathrm{i}+1, \mathrm{i}+2)=1$;

$\mathrm{DD}(\mathrm{i}+2, \mathrm{i}+1)=1$;

end

$\mathrm{DD}(1,2)=2$;

$\mathrm{DD}(2,1)=2$;

$\mathrm{DD}(\mathrm{n}-1, \mathrm{n})=2$;

$\mathrm{DD}(\mathrm{n}, \mathrm{n}-1)=2$;

DD

Eigenvaluesofpathgraph=eig(DD)

DDE $=$ sum(abs(Eigenvaluesofpathgraph))

OUTPUT:

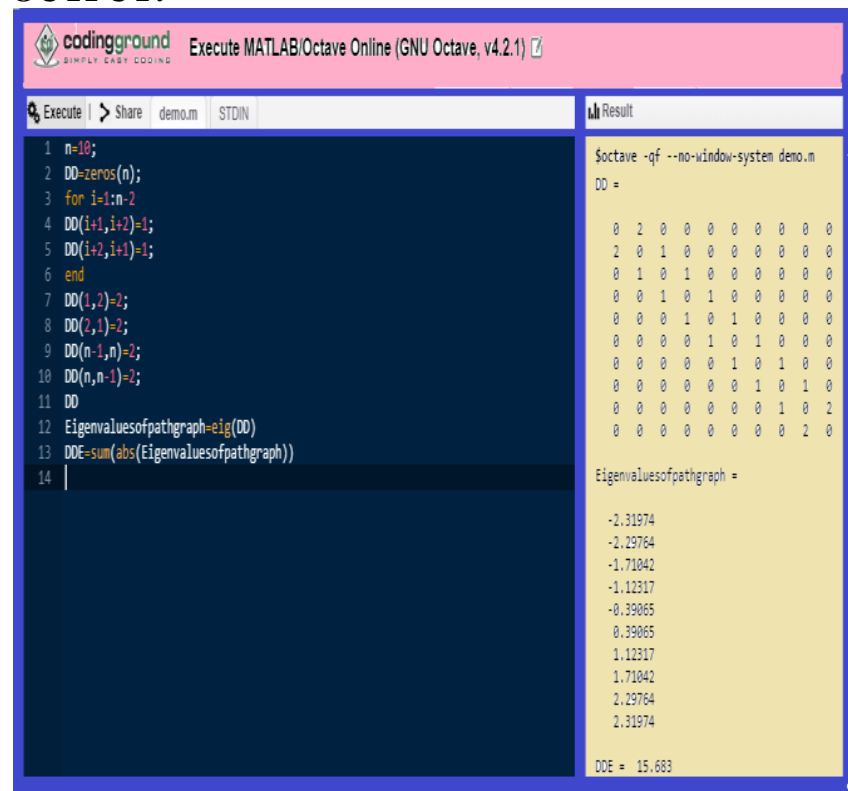

Figure 8: For $n=10$, DDE of path graph.

Published By: 
Program 2.9. To calculate the divisor degree energy of star graph:

$\mathrm{n}=; \%$ Enter the number of vertices of star graph

$\mathrm{DD}=\mathrm{zeros}(\mathrm{n})$;

for $\mathrm{i}=1: \mathrm{n}-1$

$\mathrm{DD}(\mathrm{i}, \mathrm{i}+1)=0$;

$\mathrm{DD}(\mathrm{i}+1, \mathrm{i})=0$;

$\mathrm{DD}(\mathrm{i}, \mathrm{n})=(\mathrm{n}-1)$;

$\operatorname{DD}(n, i)=(n-1)$;

end

$\mathrm{DD}(1, \mathrm{n}-1)=0$;

$\mathrm{DD}(\mathrm{n}-1,1)=0$;

DD

Eigenvaluesofstargraph=eig(DD)

$\mathrm{DDE}=\operatorname{sum}($ abs(Eigenvaluesofstargraph $))$

\section{OUTPUT:}

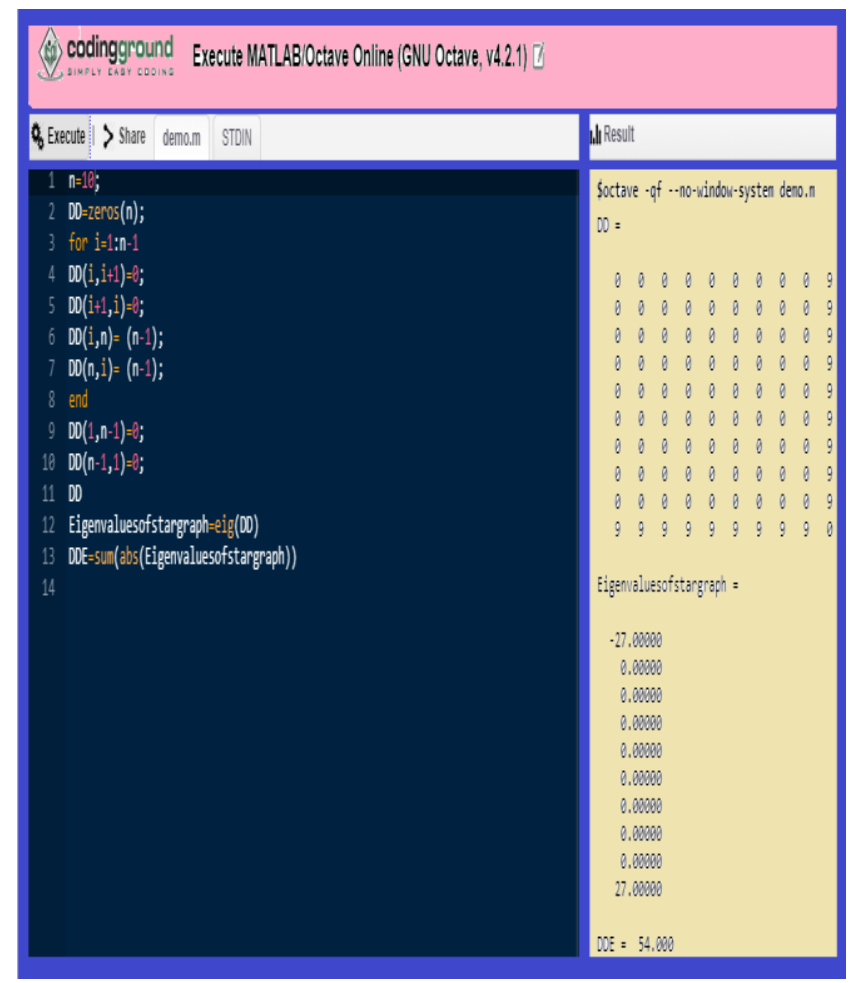

Figure 9: For $n=10$, DDE of star graph

Program 2.10. To calculate the divisor degree energy of comb graph:

$\mathrm{p}=; \%$ Enter the number of vertices of path $\operatorname{graph}(\mathrm{p}>2)$

$\mathrm{n}=2 * \mathrm{p}$;

$\mathrm{DD}=\mathrm{zeros}(\mathrm{n})$;

for $\mathrm{i}=1: \mathrm{p}-2$

$\operatorname{DD}(\mathrm{i}, \mathrm{i}+1)=1$;

$\mathrm{DD}(\mathrm{i}+1, \mathrm{i})=1$;

$\mathrm{DD}(\mathrm{i}+1, \mathrm{i}+\mathrm{n}-\mathrm{p}+1)=3$;

$\mathrm{DD}(\mathrm{i}+\mathrm{n}-\mathrm{p}+1, \mathrm{i}+1)=3$;

end

$\mathrm{DD}(1, \mathrm{p}+1)=2$;

$\mathrm{DD}(\mathrm{p}+1,1)=2$;

$\mathrm{DD}(\mathrm{p}-1, \mathrm{p})=1$;

$\mathrm{DD}(\mathrm{p}, \mathrm{p}-1)=1$;

$\mathrm{DD}(\mathrm{p}, \mathrm{n})=2$;

$\mathrm{DD}(\mathrm{n}, \mathrm{p})=2$;

DD

Eigenvaluesofcombgraph=eig(DD)

$\mathrm{DDE}=\operatorname{sum}(\operatorname{abs}($ Eigenvaluesofcombgraph $))$

\section{OUTPUT:}

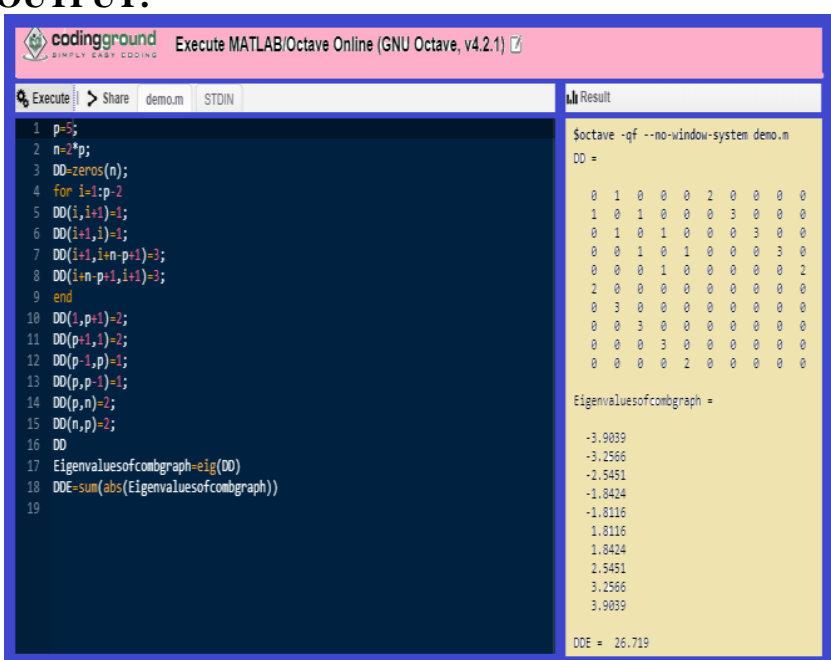

Figure 10: For $n=10$, DDE of comb graph.

The following table gives the divisor degree energy of some graphs of various orders.

TABLE I. The divisor degree energy of some graphs of order less than 20.

\begin{tabular}{|c|c|c|c|c|c|c|c|}
\hline $\begin{array}{l}\mathrm{W} \\
\mathrm{n}\end{array}$ & $\begin{array}{l}\mathrm{EDD}( \\
\left.\mathrm{W}_{\mathrm{n}}\right)\end{array}$ & $\mathrm{F}_{\mathrm{n}}$ & $\begin{array}{l}\text { EDD } \\
\left(F_{n}\right)\end{array}$ & $\mathrm{K}_{\mathrm{p}, \mathrm{q}}$ & $\begin{array}{l}\text { EDD } \\
\left(K_{\mathrm{p}, \mathrm{q}}\right)\end{array}$ & $\begin{array}{l}\mathbf{S}\left(\mathrm{K}_{1, \mathrm{n}}\right. \\
-1)\end{array}$ & $\begin{array}{l}\text { EDD } \\
\left(\mathrm{S}^{\prime}\left(\mathrm{K}_{1, \mathrm{n}-1}\right)\right)\end{array}$ \\
\hline $\begin{array}{l}\text { W } \\
4\end{array}$ & 6 & $\mathrm{~F}$ & $\begin{array}{l}5.12 \\
3\end{array}$ & $\begin{array}{l}\mathrm{K}_{2}, \\
2\end{array}$ & 4 & $\begin{array}{l}\mathbf{S}^{\prime}(\mathrm{K} \\
1,2)\end{array}$ & 15.232 \\
\hline $\begin{array}{l}\text { W } \\
5\end{array}$ & $\begin{array}{l}6.47 \\
2\end{array}$ & $\mathrm{~F}$ & $\begin{array}{l}8.94 \\
4\end{array}$ & $\begin{array}{l}\mathrm{K}_{2}, \\
3\end{array}$ & 4.899 & $\begin{array}{l}\mathrm{S}^{\prime}(\mathrm{K} \\
1,3)\end{array}$ & 26.382 \\
\hline $\begin{array}{l}\text { W } \\
6\end{array}$ & $\begin{array}{l}9.37 \\
1\end{array}$ & $\mathrm{~F}$ & $\begin{array}{l}11.0 \\
6\end{array}$ & $\begin{array}{c}\mathrm{K}_{2}, \\
4\end{array}$ & 11.31 & $\begin{array}{l}\mathrm{S}^{\prime}(\mathrm{K} \\
1,4)\end{array}$ & 43.081 \\
\hline $\begin{array}{l}\text { W } \\
7\end{array}$ & 16 & $\mathrm{~F}$ & $\begin{array}{l}17.1 \\
3\end{array}$ & $\begin{array}{c}\mathrm{K}_{3}, \\
4\end{array}$ & 6.928 & $\begin{array}{l}S^{\prime}(K \\
1,5)\end{array}$ & 58.138 \\
\hline $\begin{array}{l}\text { W } \\
8\end{array}$ & $\begin{array}{l}17.7 \\
5\end{array}$ & $\begin{array}{l}\mathrm{F} \\
8\end{array}$ & $\begin{array}{l}18.7 \\
1\end{array}$ & $\begin{array}{c}\mathrm{K}_{4}, \\
4\end{array}$ & 8 & $\begin{array}{l}S^{\prime}(K \\
1,6)\end{array}$ & 79.145 \\
\hline $\begin{array}{l}\text { W } \\
9\end{array}$ & $\begin{array}{l}19.1 \\
4\end{array}$ & $\mathrm{~F}$ & $\begin{array}{l}22.6 \\
1\end{array}$ & $\begin{array}{l}\mathrm{K}_{4}, \\
5\end{array}$ & 8.944 & $\begin{array}{l}\mathrm{S}^{\prime}(\mathrm{K} \\
1,7)\end{array}$ & 97.283 \\
\hline $\begin{array}{l}\text { W } \\
10\end{array}$ & $\begin{array}{l}27.6 \\
2\end{array}$ & 10 & $\begin{array}{l}28.4 \\
5\end{array}$ & $\begin{array}{c}\mathrm{K}_{3}, \\
7\end{array}$ & 18.33 & $\begin{array}{l}\mathrm{S}^{\prime}(\mathrm{K} \\
1,8)\end{array}$ & 121.85 \\
\hline
\end{tabular}

TABLE II. The divisor degree energy of tree graphs of order up to 10 .

\begin{tabular}{|l|l|l|l|l|l|}
\hline $\mathrm{P}_{\mathrm{n}}$ & $\operatorname{EDD}\left(\mathrm{P}_{\mathrm{n}}\right)$ & $\mathrm{S}_{\mathrm{n}}$ & $\operatorname{EDD}\left(\mathrm{S}_{\mathrm{n})}\right.$ & $\mathrm{P}_{\mathrm{n}-1}{ }^{\circ} \mathrm{K}_{1}$ & $\operatorname{EDD}\left(\mathrm{P}_{\mathrm{n}-1}{ }^{\circ} \mathrm{K}_{1}\right)$ \\
\hline $\mathrm{P}_{4}$ & 8.2462 & $\mathrm{~S}_{4}$ & 10.392 & $\mathrm{P}_{3}{ }^{\circ} \mathrm{K}_{1}$ & 20.556 \\
\hline $\mathrm{P}_{5}$ & 8.8990 & $\mathrm{~S}_{5}$ & 16 & $\mathrm{P}_{4} \mathrm{~K}_{1}$ & 26.719 \\
\hline $\mathrm{P}_{6}$ & 10.657 & $\mathrm{~S}_{6}$ & 22.361 & $\mathrm{P}_{5} \mathrm{~K}_{1}$ & 32.883 \\
\hline $\mathrm{P}_{7}$ & 11.587 & $\mathrm{~S}_{7}$ & 29.394 & $\mathrm{P}_{6}{ }^{\circ} \mathrm{K}_{1}$ & 39.046 \\
\hline $\mathrm{P}_{8}$ & 13.159 & $\mathrm{~S}_{8}$ & 37.041 & $\mathrm{P}_{7} \mathrm{~K}_{1}$ & 45.209 \\
\hline $\mathrm{P}_{9}$ & 14.194 & $\mathrm{~S}_{9}$ & 45.255 & $\mathrm{P}_{8} \mathrm{~K}_{1}$ & 51.373 \\
\hline $\mathrm{P}_{10}$ & 15.683 & $\mathrm{~S}_{10}$ & 54 & $\mathrm{P}_{9} \mathrm{~K}_{1}$ & 57.536 \\
\hline
\end{tabular}

Published By: 


\section{REFERENCES}

[1] R. Balakrishnan, "The energy of a graph", Linear Algebra and its Appl. 387 (2004) 287-295.

[2] Bondy.J.A, Murty.U.S.R, Graph Theory with Application, MacMilllan, New York, 1976.

[3] I. Gutman, "The Energy of a Graph", Ber. Math. Stat. Sekt. Forschungszent Graz 103 (1978) 1-22.

[4] S. P. Kanniga Devi and K. Nagarajan, "Divisor Degree Energy of Graphs", Int. J. Math. Archive 8 (2017) 29-35.

[5] Xueliang Li, Yongtang Shi, Ivan Gutman, "Graph Energy”, Springer, New York, 2012.

\section{AUTHORS PROFILE}

Dr. K. Nagarajan is working as a Professor of mathematics at Kalasalingamuniversity, Krishnankoil. He was working as Head and Associate Professor of Department of mathematics at Sri S.Ramasamy Naidu Memorial College, Sattur. He has guided 38 M.Phil. Scholars and 4 $\mathrm{Ph} . \mathrm{D}$. Scholars. He is currently guiding $5 \mathrm{Ph} . \mathrm{D}$. Scholars. Having published 11 research papers in national level journals and 40 research papers in international level journals.He has completed Minor Research Project sponsored by UGC.He has 9 years experience in research and his area of interests in research is graph labelling, domination of graphs, distances in graphs and energy of graphs.

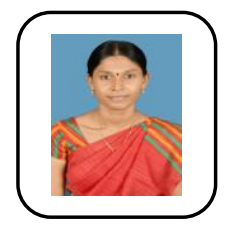

S. P. Kanniga Devi is a Research Scholar of department of mathematics in Sri S.Ramasamy Naidu Memorial College, Sattur. She is pursuing her Ph.D. in Divisor Degree Energy of Graphs in Graph theory under the guidance of Dr.K.Nagarajan. She has completed her UG and PG degree at S.F.R.College, Sivakasi and M.Phil. Degree at Sri S.Ramasamy Naidu Memorial College, Sattur. Her research work is concerned with the Energy of Graphs. 